\title{
Reduced-Order Modeling of Compressible Flows with Unsteady Normal Shock Motion
}

\author{
Christopher D. Marley* and Karthik Duraisamy ${ }^{\dagger}$ \\ University of Michigan, Ann Arbor, Michigan, 48109, U.S.A.
}

\begin{abstract}
Projection-based reduced-order modeling has been used successfully to develop efficient models of fluid flows. However, a majority of the applications are limited to small perturbations about a nominal flow condition and do not typically address strong nonlinearities. In the present work, we assess the viability of reduced-order modeling to the problem of unstart in high-speed engine inlets. A complicating factor in this application is the presence of strong shock waves. Models based on a linearized flow assumption fail to capture the large shock motions associated with unstart. Projection-based, Proper Orthogonal Decomposition (POD) models can - in theory - account for such nonlinearities, but at a very high cost. Advances have been made recently in developing techniques to further accelerate projection-based models. One such method, the Discrete Empirical Interpolation Method (DEIM), is applied in this study to two nozzle flow cases: a fully-expanded nozzle and a nozzle with a normal shock present. In both examples, the model is based on a quasi-one-dimensional inviscid flow assumption. This study highlights the lack of robustness of the DEIM and proposes an alternative acceleration method based on L2-norm minimization. For both test cases, instances are found where DEIM is unstable but L2norm minimization is not, motivating further work in nonlinear acceleration techniques employing optimization, including L2-norm minimization and compressed sensing.
\end{abstract}

\section{Introduction}

Supersonic and hypersonic air-breathing engines are susceptible to a phenomenon referred to as unstart. Unstart can be instigated by occurrence of thermal choking, flow separation or imperfect stoichiometry and is characterized by a breakdown of supersonic flow in the system. During unstart of a high-speed inlet, the shock system inside the inlet propagates forward and become dislodged. Unstart leads to a sudden loss in thrust and a redistribution of external aerodynamic forces, causing drastic changes in vehicle dynamics. Engine unstart is a common issue in many air-breathing systems and much energy and resources have been expended to better understand and prevent it. ${ }^{1}$

With the ever-increasing demand for safe, efficient and versatile aircraft, designers seek to model more complex phenomena, such as engine unstart, in the early vehicle design stages. Computationally inexpensive models are required at the preliminary design stage of high-speed vehicles that are robust with respect to unstart and for control design during unstart. Flow complexity, however, presents a major challenge in modeling high-speed inlets. High-speed inlet flows are characterized by shock wave-boundary-layer interactions, large shock motion, three-dimensional flow and high turbulence. ${ }^{2}$ Modeling these flow phenomena is computationally intensive.

Recent efforts at the Air Force Research Lab at Wright Patterson AFB have focused on design and control-oriented modeling of hypersonic vehicles. The Bolender-Doman model employs physics-based, lowfidelity models to simulate a generic waverider-type hypersonic aircraft. ${ }^{3}$ The Bolender-Doman model is a first-principles reduced-fidelity model. Since the vehicle is slender, conventional piston theory was used to compute aerodynamic forces. The engine flow was assumed to be one-dimensional, the bow shock was assumed to be two-dimensional, and any shock waves downstream of the bow shock were ignored. Researchers have improved the Bolender-Doman model in order to avoid some of the assumptions that were made

\footnotetext{
*Graduate Research Assistant, Department of Aerospace Engineering, AIAA Member, cmarley@umich.edu.

$\dagger$ Assistant Professor, Department of Aerospace Engineering, AIAA Fellow, kdur@umich.edu.
} 
originally. ${ }^{4-7}$ In particular, the Bolender-Doman model is modified to compute both the engine unstart and the ram-scram transition boundaries for a trimmed waverider vehicle. ${ }^{6}$

A major assumption in the Bolender-Doman model and its derivatives is the use of steady models. Any dynamics associated with unstart are only quasi-steady. As a result, the unstart limits on the flight envelope predict only the beginning stages of unstart based on a perceived safe shock train location. ${ }^{8}$ In reality, an unstart can occur even before this designated safe-zone if the dynamics perturbing the shock location (i.e. gusts, vehicle acceleration, fuel throttling) are severe. An unsteady model capable of simulating strong shocks undergoing large oscillations is required. This research applies the recent developments in reducedorder modeling of compressible flows ${ }^{9,10}$ to several example cases to better understand the challenges in reduced-order representations of strong shock waves undergoing large shock motions.

This present work focuses on POD/Galerkin projection-based reduced-order modeling of fully-nonlinear flows using the Discrete Empirical Interpolation Method ${ }^{11}$ (DEIM) and L2-Norm Minimization for acceleration. Projection-based ROMs are briefly discussed in Section II. One limiting factor in employing conventional POD/Galerkin projection is the presence of nonlinear residual terms, which must still be computed at every grid point during each time iteration, negating any computational speed-up. To circumvent this limitation, the residual terms are estimated using only a small sample of grid points. DEIM and L2-Norm Minimization are both methods of estimating the full residual and are detailed in Section III. Both DEIM and L2-Norm Minimization are then applied to an unsteady, fully-expanded nozzle flow in Section IV and an unsteady nozzle flow with a normal shock in Section V. Stability aspects of DEIM and L2-Norm Minimization will be discussed in particular. Finally, this paper ends with a brief summary of POD/Galerkin projection-based ROMs and how L2-Norm Minimization can be utilized to improve stability over DEIM for nonlinear acceleration.

\section{Projection-Based Reduced-Order Modeling}

Different approaches exist to obtain unsteady reduced-order models. One class of modeling is empirical based where system identification techniques are used to relate the system input to some observed output. For example, in Ref. 12 Hutzel et al. model the unsteady leading edge location of a shock-train assuming a nonlinear model structure and then vary the back pressure during experiments to obtain a model. Another approach is physics-based, reduced fidelity modeling where the fundamental conservation equations are solved, but certain assumptions are made in order to reduce the complexity of the problem (as in the Bolender-Doman model described earlier). Early attempts at reduced-fidelity modeling of unsteady inlets employ the normal shock relations and model acoustic waves which perturb the flow and simulate shock motion. ${ }^{13}$ Another popular approach is to use linearized Euler/Navier-Stokes equations. ${ }^{14,15}$ Linearized models however, apply only to one design point; for the purpose of this research, a nonlinear model capable of simulating the inlet over a range of conditions is desired.

POD/Galerkin projection-based model reduction techniques can capture both linear and nonlinear models of compressible flows with unsteady shock motion. ${ }^{9,10}$ In the POD/Galerkin projection method, proper orthogonal decomposition is used to form a reduced-order basis. Holmes et al. discuss POD in detail in Ref. 16. When velocity flow variables are of primary interest, the reduced-order POD-modes are optimal in the sense that, using an $L_{2}$ inner product, the modes capture the greatest amount of average kinetic energy of the flow. However, for flow variables important in highly compressible flow (i.e. density and temperature), the $L_{2}$ inner product makes little physical sense and alternative inner products have been proposed. ${ }^{17}$ After the POD bases are formed, a Galerkin projection is preformed where the governing equations are projected onto the POD bases forming a set of coupled, nonlinear ordinary differential equations in time.

The first step in projection-based reduced-order modeling is to develop a reduced basis which can approximately reproduce the full-order solution. Proper orthogonal decomposition is a popular method to develop this basis and is employed here. Consider a general form of the unsteady, quasi-one-dimensional Euler equations:

$$
\frac{\partial U(x, t)}{\partial t}=R(U(x, t))
$$

where $U=\left[\rho, \rho u, \rho e_{t}\right]^{T}$ and $R$, the residual vector, is a nonlinear function of $U$ :

$$
R=-\frac{\partial F(x, t)}{\partial x}+Q(x, t)
$$


where $F$ is the flux vector $\left(F=\left[\rho u, \rho u^{2}+p, \rho h_{t} u\right]^{T}\right)$ and $Q$ is the source vector $\left(Q=[0, p d A / d x, 0]^{T}\right)$.

For the full-order model, the order of the system, $N$, is defined as the number of grid cells, $N_{C}$, times the number of conservation variables (three in this case: $U_{1}=\rho, U_{2}=\rho u, U_{3}=\rho e_{t}$ ). Snapshots of the flow solution are compiled into a matrix $\mathbf{S}$ on which a singular value decomposition is then performed to generate the POD reduced-order bases: $\phi_{j}, j=1,2, . ., M_{L}$ where $M_{L}$ is the number of basis vectors the reduced-order model is truncated to. ${ }^{10,16}$ Each of the $\phi_{j}$ basis vectors is a $N \times 1$ column vector. The bases containing the $M_{L}$ largest energies, where $M_{L} \ll N$, are selected for the reduced-order model. The remaining basis vectors (those containing the small energies) are neglected. The POD basis vectors are used to approximate the full-order solution as:

$$
U(x, t)=\sum_{j=1}^{M_{L}} a_{j}(t) \phi_{j}(x)
$$

where $a_{j}(t)$ are the POD coefficients which vary with time only; the POD basis vectors $\phi_{j}(x)$ account for the spatial dependence. A set of $M_{L}$ ordinary differential equations for $a_{j}(t)$ is obtained by projecting the governing equations onto the POD bases as follows:

$$
\left(\left[\frac{\partial}{\partial t}-R\right] \sum_{i=1}^{M_{L}} a_{i}(t) \phi_{i}(x), \phi_{j}\right)=0 ; j=1,2, \ldots M_{L}
$$

where $(\cdot, \cdot)$ is the $L_{2}$ inner product. The result, after performing the inner product and multiplying by $\Phi^{T}$, is a set of $M_{L}$ ordinary differential equations of the form:

$$
\frac{\partial}{\partial t} a(t)_{M_{L} \times 1}=\Phi_{M_{L} \times N}^{T} R(\Phi a(t))_{N \times 1}
$$

where $\Phi$ is the matrix form of the POD basis vectors: $\Phi_{N \times M_{L}}=\left[\phi_{1_{N \times 1}}, \phi_{2_{N \times 1}}, \ldots, \phi_{M_{L N \times 1}}\right]$.

\section{Acceleration of Nonlinear Flows}

When the residual term, $R(x, t)$, is nonlinear, Eq. (5) requires a full function evaluation at each time step; therefore, while the number of equations to solve has been significantly reduced (from $N$ equations to $M_{L}$ equations), the actually computational time has not. One approach to reduce the amount of computations is to calculate only a sample of the residual terms and, using an appropriate basis for the residual space, use these sample residuals to reconstruct the full residual. The discrete empirical interpolation method ${ }^{11}$ is one such approach. Another method, based of optimization techniques and referred to as L2-Norm Minimization, is proposed. Both of these methods are discussed in the current section.

\section{A. Discrete Empirical Interpolation Method}

In the discrete empirical interpolation method, the residual term is represented as a linear combination of POD basis vectors, similar to Eq. (3), only using the residual space instead of the solution space to form the reduced bases:

$$
R_{N \times 1}=\Psi_{N \times M_{L}} c_{M_{L} \times 1}
$$

where $\Psi$ is the matrix containing the reduced bases of the residual space, $c$ is the vector of POD coefficients and $M_{L}$ is the number of POD bases selected. Premultiplying Eq. (6) by $P^{T}$ (where $P$ is a $N \times M_{L}$ matrix with values of one at the $M_{L}$ sample point locations and zero everywhere else) and solving for the POD coefficients, $c$, results in the following equation for the full residual:

$$
R_{N \times 1}=D_{N \times M_{L}} \hat{R}_{M_{L} \times 1}
$$

where $R(t)$ is the full residual, $\hat{R}(t)$ is the residual sampled at $M_{L}$ points and the matrix $D$ is precomputed as:

$$
D_{N \times M_{L}}=\Psi_{N \times M_{L}}\left(P^{T} \Psi\right)_{M_{L} \times M_{L}}^{-1}
$$

Knowing which points to sample is critical; the discrete empirical interpolation method provides an algorithm for selecting the sample points. ${ }^{11}$ The DEIM algorithm selects the residual sampling locations 
based solely on the residual basis vectors $\Psi$. This is one of DEIM's limitations; selecting alternative sampling locations requires selecting different bases. Another limitation of DEIM is that, once the basis vectors have been selected to form the reduced-order model, the sampling locations are then fixed in time.

\section{B. L2-Norm Minimization}

The DEIM has been shown to be very effective in many problems, but can be problematic in flows with discontinuities as it matches the residual at every sampling location, and thus it has a tendency to overfit. We propose L2-Norm Minimization as an alternative technique. In L2-Norm Minimization, selection of the sampling point locations is arbitrary. After the residuals are calculated at the selected sampling locations, optimization techniques are used to reconstruct the full residual. Unlike DEIM, the number of sampling points, $L$, does not depend on the number of basis vectors, $M_{L}$. In L2-Norm Minimization, the following system of equations is solved:

$$
(P \Psi)_{L \times M_{L}} b_{M_{L} \times 1}=\hat{R}_{L \times 1}
$$

where $\hat{R}$ is the vector of residuals calculated at $L$ sample locations, $P$ is an $L \times N$ matrix of zeros everywhere except for points corresponding to the sampling locations where the value in the matrix is one and $M_{L}$ is the number of POD basis vectors. Equation (9) is solved for the coefficients, $b$, in an L2-norm sense:

$$
\min _{b=\left(b_{1}, \ldots, b_{M_{L}}\right)}\|(P \Psi) b-\hat{R}\|^{2}
$$

Note that this method has similarities to the Gappy POD ${ }^{18}$ especially if the same sample locations are chosen. However, we use a Levenberg-Marquardt algorithm to solve the above equation thus numerical results will be different for nonlinear problems. Once the $b$ coefficients are computed, they will be used to calculate the full residual as:

$$
R_{N \times 1}=\Psi_{N \times M_{L}} b_{M_{L} \times 1}
$$

\section{Perfectly-Expanded Nozzle Flow}

In order to illustrate the POD/Galerkin projection-based reduced-order modeling technique, and to assess the method's stability and effectiveness when applied to fully-nonlinear flows, flow through a convergingdiverging nozzle is analyzed. Also assessed are the DEIM and L2-Norm Minimization acceleration techniques. The nozzle contour, shown in Fig. 1, is based on the experimental nozzle in Ref. 19.

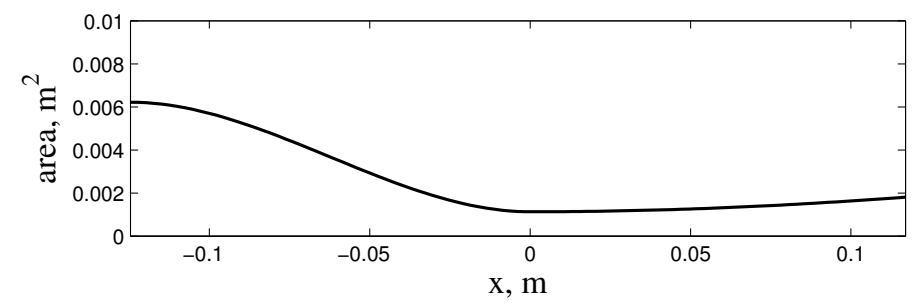

Figure 1: Nozzle area distribution (nozzle throat at $\mathrm{x}=0$ ).

The full-order solution is obtained by solving Eq. (1) using the finite volume technique. The numerical flux is evaluated using kinetic flux vector splitting ${ }^{20}$ and a multi-stage Runge-Kutta is used for temporal discretization. To simulate a perfectly expanded nozzle, a supersonic outflow boundary condition is imposed along with a subsonic inflow boundary condition with specified inlet stagnation pressure $p_{t, i}$. The simulations in this section are performed on a grid of 100 evenly spaced cells. To obtain unsteady solutions in time, the inlet stagnation pressure is gradually increased from $5 \mathrm{~atm}$ to $10 \mathrm{~atm}$ over 0.01 seconds.

The conservation variables from the unsteady full-order solution are compiled into a snapshot matrix $\mathbf{S}$ every 100 iterations from the initial time until a new steady-state is reached. An SVD is then preformed on 
the snapshot matrix, the results of which can be seen in Fig. 2a. Note that the first mode is dominant; the singular value of the first mode is four orders of magnitude larger than the second mode, implying that the modes with smaller energy content are negligible. The basis vectors of the first three modes are shown in Fig. 2b.

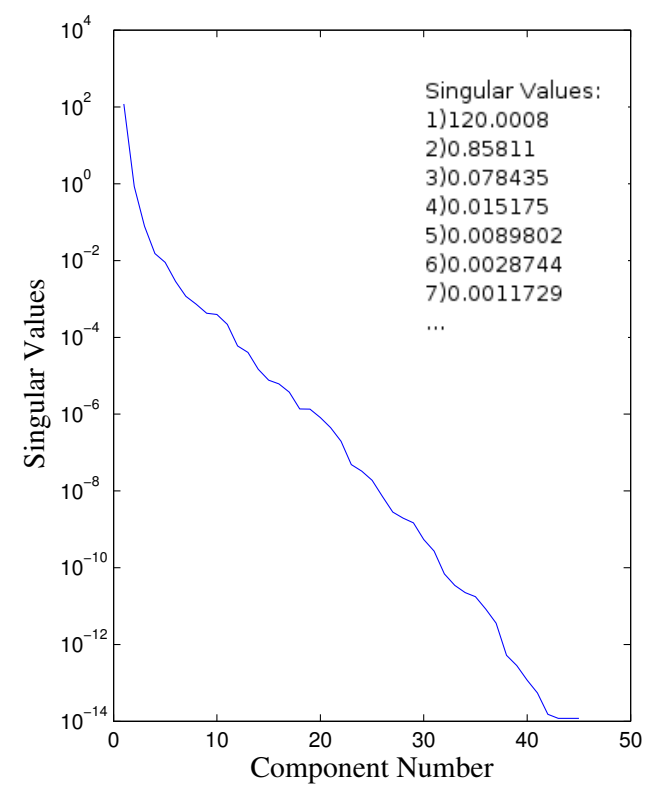

(a)
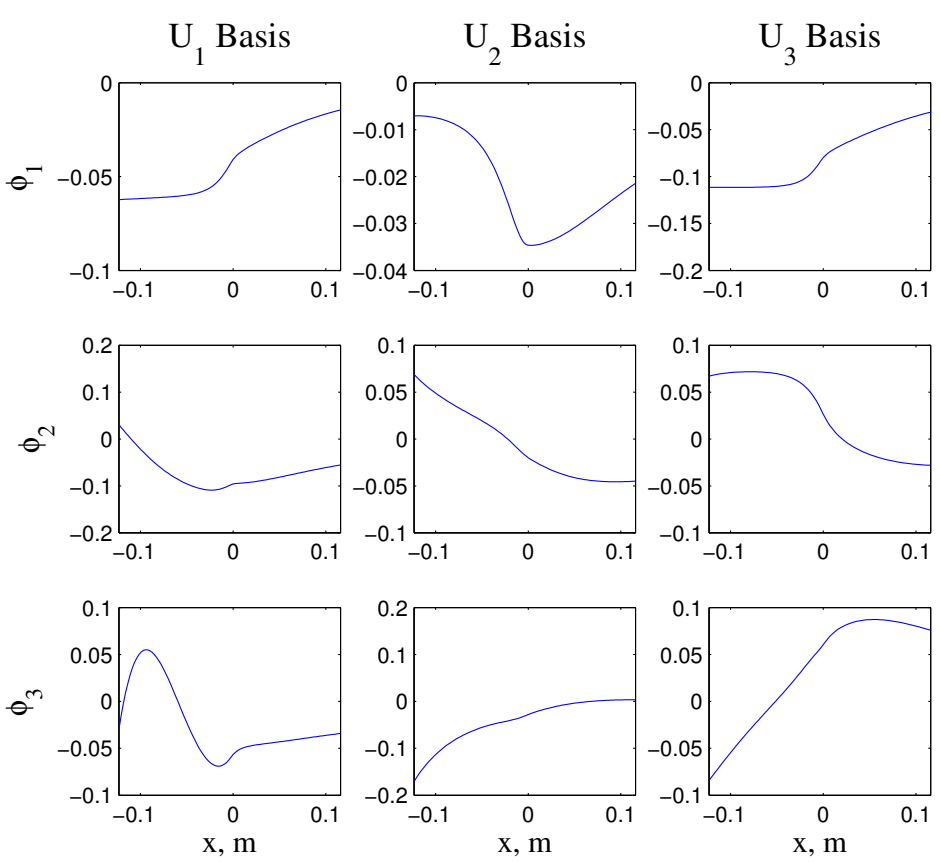

(b)

Figure 2: (a) Singular values of snapshot matrix for the unsteady ramp increase from $p_{t, i}=$ 5 to 10 atm over 0.01 seconds. (b) First three basis vectors $\left(\phi_{1}, \phi_{2}, \phi_{3}\right)$ as a function of $x$ for each of the three conservation variables $\left(U_{1}, U_{2}, U_{3}\right)$ for the unsteady ramp increase from $p_{t, i}$ $=5$ to 10 atm over 0.01 seconds.

Figure 3 displays the solution to Eq. (5) for the POD coefficients, $a_{j}, j=1, \ldots, M_{L}$, versus time using three basis vectors $\left(M_{L}=3\right)$ with an increased time step $(\mathrm{CFL}=40)$. Also shown in Fig. 3 are the corresponding POD coefficients required to reproduce the exact solution from the full-order model for comparison. The magnitudes of the POD coefficients confirm that the first mode is indeed dominant. While there is a noticeable error in the second and third modes when compared to the full-order solution, only a small error is observed in the first mode. Figure 4 shows pressure (reconstructed from the reduced-order model POD coefficients using Eq. (3)) versus time at four locations in the nozzle. Pressure from the full-order solution is also shown in the figure. Note that the error in the second and third modes observed in Fig. 3 does not manifest itself in the pressure values reconstructed from the POD coefficients. The same is true for the other two primitive variables $\rho$ and $u$. 

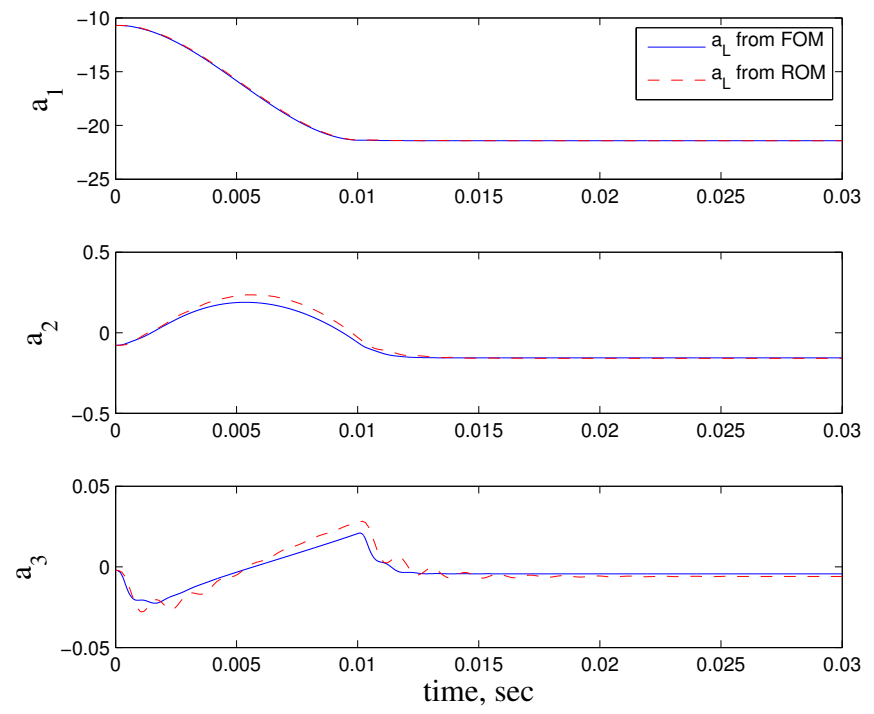

Figure 3: POD coefficients $\left(a_{1}, a_{2}, a_{3}\right)$ versus time from the reduced-order model (using three basis vectors and increasing CFL to 40 ) along with the corresponding POD coefficients required to reproduce the full-order solution for comparison.
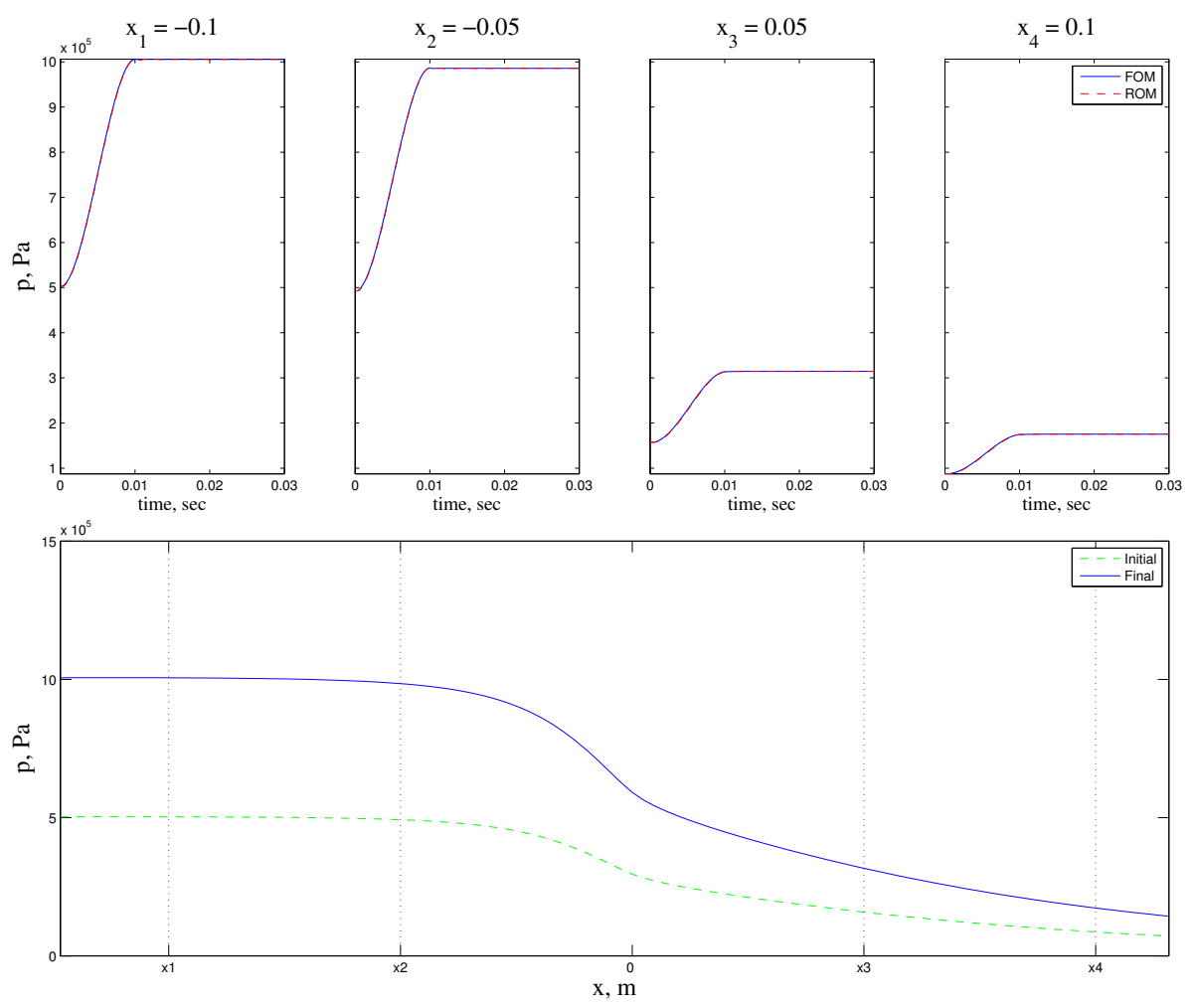

Figure 4: Pressure (reconstructed from the reduced-order model POD coefficients) versus time at four locations in the nozzle along with pressure from the full-order solution for comparison. 
One reason why POD/Galerkin projection-based reduced-order modeling is effective in decreasing the computational time required to perform an unsteady simulation, is the ability to increase the time step to a point where it would otherwise become unstable in the full-order model. Equation (5), the reduced-order system, models the POD coefficients in time and has different stability properties compared to Eq. (1) for the full-order system which models the conservation variables. The results in Figs. 3 and 4 , for example, are obtained at a CFL of 40 whereas the corresponding full-order model was obtained using a CFL of 2.5, the maximum stable value for the four-stage RK method used. The resulting speed-up factor in this case is 17 . The reason why the increased time step is possible is that the small scale modes, where instabilities often first manifest themselves, are neglected. There is still a limit to the increased time step; looking at Fig. 3 closely reveals spurious oscillations in the third mode which eventually subside. The instability can clearly be seen, however, in Fig. 5 where the CFL is increased to 80 .
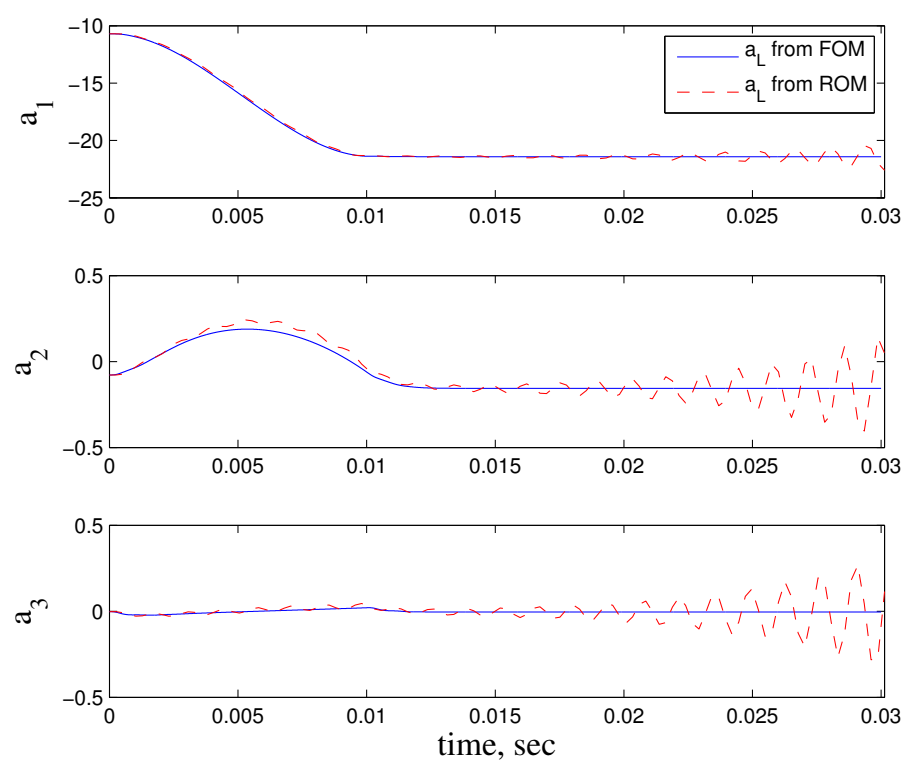

Figure 5: POD coefficients $\left(a_{1}, a_{2}, a_{3}\right)$ versus time from the reduced-order model (using three basis vectors and increasing CFL to 80 ) along with the corresponding POD coefficients required to reproduce the full-order solution for comparison.

As mentioned earlier, another method to achieve computational speed-up is to, instead of relying on increasing the time step, employ a nonlinear acceleration technique such as DEIM described in Part A of Section III or L2-Norm Minimization described in Part B of Section III. The first step is to form a reduced basis for the residual space. The residuals from the full-order solution are compiled into a snapshot matrix and, after performing an SVD and retaining the first $M_{L}$ modes, the residual POD bases are obtained: $\psi_{1}$, $\psi_{2}, \ldots, \psi_{M_{L}}$. Using the DEIM sample point selection algorithm, $M_{L}$ sampling location are selected. Note that according to the DEIM algorithm, the number of sampling locations must be identical to the number of basis vectors used.

While achieving acceleration through residual sampling can be successful, the technique is not always robust. Figure 6a shows the eigenvalues of the Jacobian matrix $d R(U) / d U$ for the reduced-order model using six basis vectors. The eigenvalues plotted in Fig. 6a all have negative real parts, suggesting a stable solution for the reduced-order model without acceleration. The eigenvalues in Figure 6a are also nearly identical to the eigenvalues for the full-order model. However, Fig. 6b shows the eigenvalues of the Jacobian matrix with DEIM employed for acceleration, again using six basis vectors. Note that most of the eigenvalues collapse to zero because the full residual term depends only on a few sampled residuals. Furthermore, the maximum real part of the eigenvalues is positive and the solution goes unstable. On the other hand, Fig. 7 shows the eigenvalues of the Jacobian matrix with L2-Norm Minimization employed for acceleration instead of DEIM. Note that, while most of the eigenvalues still collapse to zero, the nonzero eigenvalues all have negative real 
parts and a stable soltion is obtained.

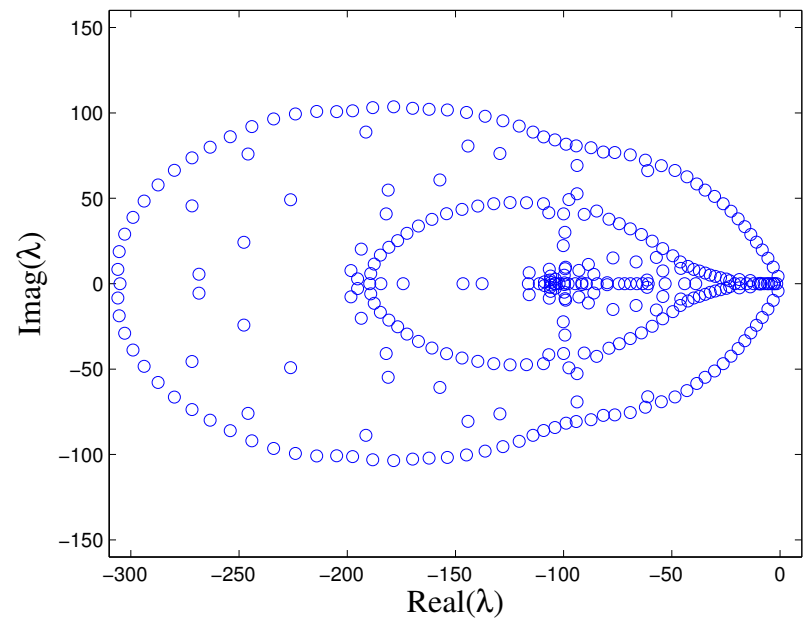

(a) Without acceleration.

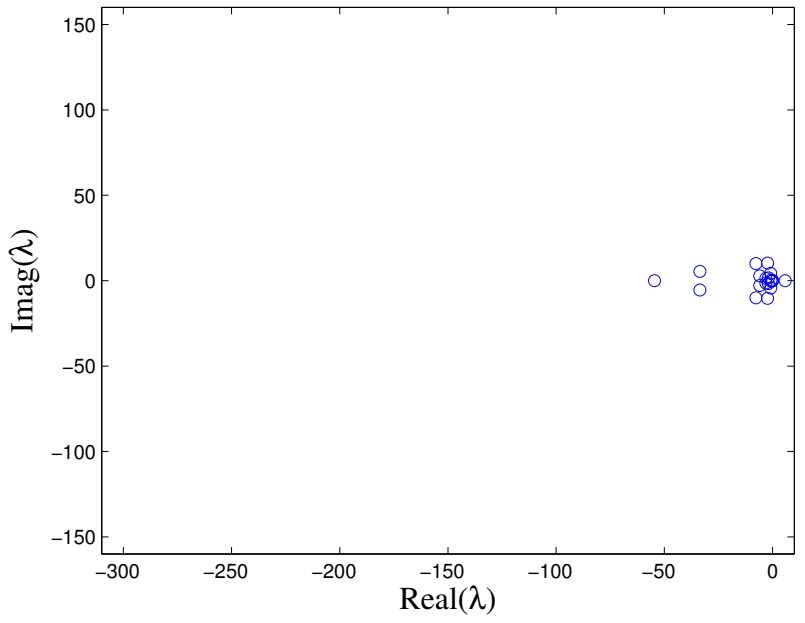

(b) Acceleration using DEIM.

Figure 6: Eigenvalues of the Jacobian matrix $d R(U) / d U$ from the reduced-order model (a) without acceleration and (b) employing DEIM for acceleration. ( $M_{L}=6$ for both cases.)

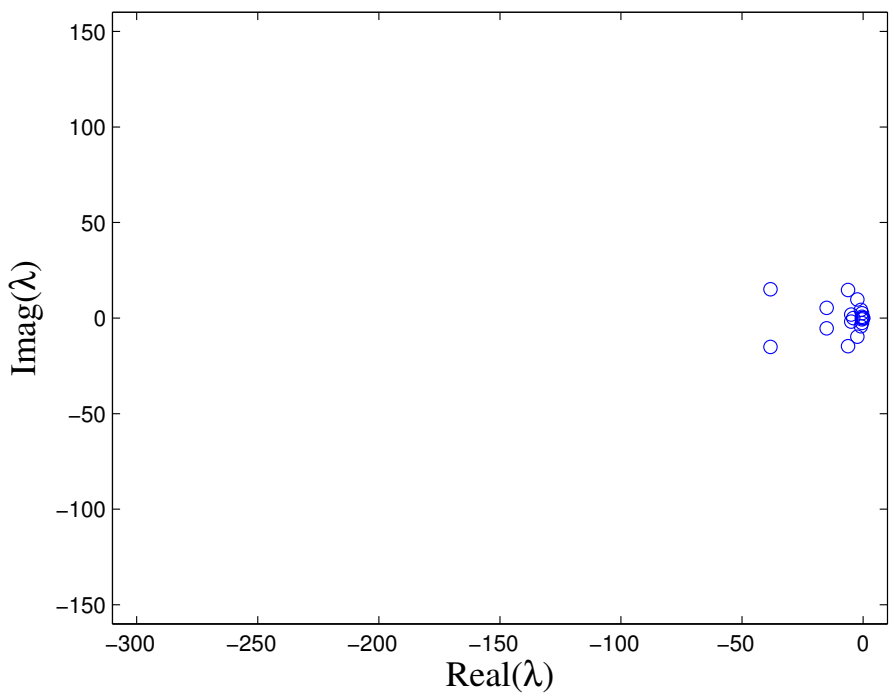

Figure 7: Eigenvalues of the Jacobian matrix $d R(U) / d U$ from the reduced-order model employing L2-Norm Minimization for acceleration and using six basis vectors.

To gain further insight into the stability of the reduced-order model, the global eigenvectors are compared for the different models. The global eigenvectors for the full-order model are identical to the global eigenvectors for the reduced-order model. Figure 8 compares the global eigenvectors of the ROM employing DEIM and L2-Norm Minimization to the global eigenvectors of the ROM without acceleration. Note that there is some improvement, particularly for the eigenvector corresponding to $U_{2}$, using L2-Norm Minimization over the conventional DEIM approach to acceleration. 

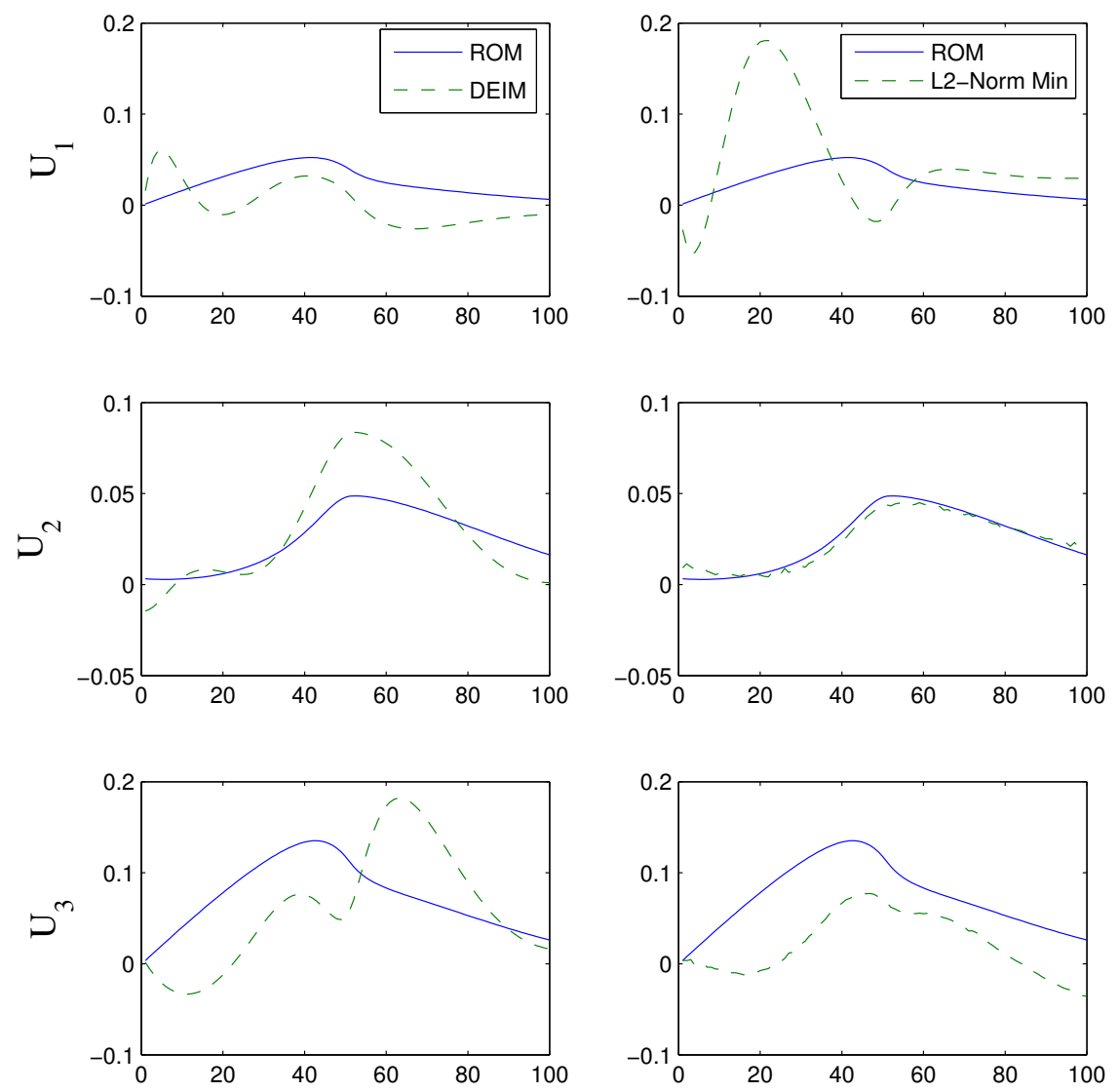

Figure 8: Global eigenvectors of the reduced-order model without acceleration compared to the reduced-order model employing DEIM acceleration in the left column and L2-Norm Minimization acceleration in the right column.

\section{Normal Shock in Diverging Section}

In this section, nozzle flow with a subsonic boundary condition at both the inlet and exit (resulting in a normal shock in the diverging section of the nozzle) is studied. Flow is established in the nozzle at an initial pressure ratio $\left(p_{e} / p_{t, i}\right)$ of 0.87 . The inlet stagnation pressure $p_{t, i}$ is maintained at a constant value of 10 atm while the exit static pressure $p_{e}$ is slowly decreased to a final pressure ratio of 0.85 over 0.01 seconds. The simulations in this section are performed on a grid of 1000 evenly spaced cells. The initial and final solutions are shown in Fig. 9.

Following the same procedure as before for developing the reduced-order model, the conservation variables $U_{1,2,3}$ from the unsteady, full-order model are compiled into a snapshot matrix and an SVD is performed. The singular values are shown in Fig. 10a. As was the case for the fully-expanded nozzle example in the previous section, the first singular value is dominant. However, the drop-off in the singular values is not as significant, indicating that more bases are required to reconstruct a flowfield with shocks. Figure 10b shows the first four basis vectors resulting from the SVD. 

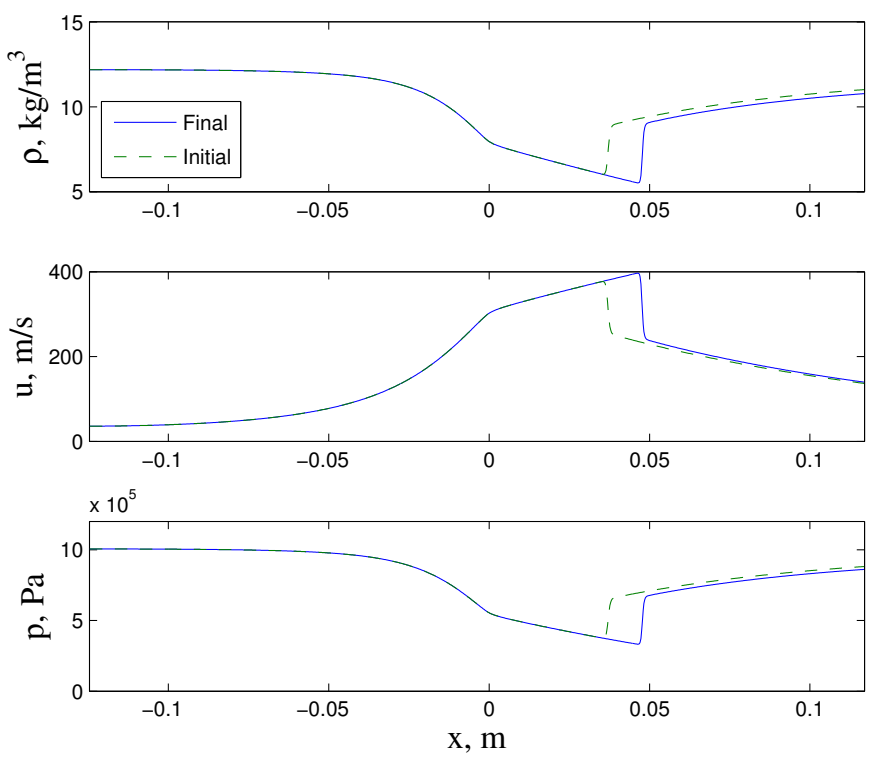

Figure 9: Solution to case with normal shock in diverging section of the nozzle. Initial solution is at $p_{e} / p_{t, i}=\mathbf{0 . 8 7}$ and the final solution is at $p_{e} / p_{t, i}=\mathbf{0 . 8 5}$

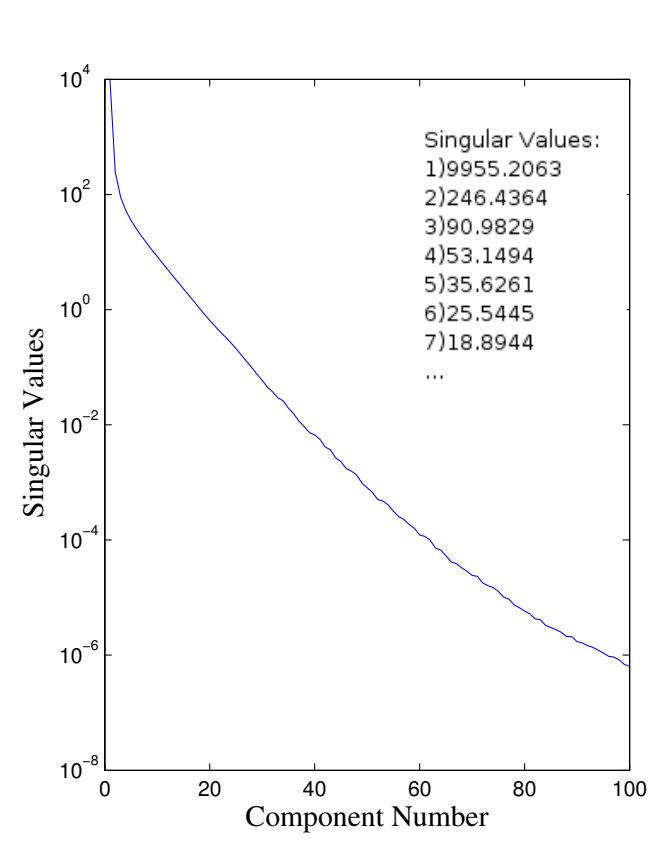

(a)
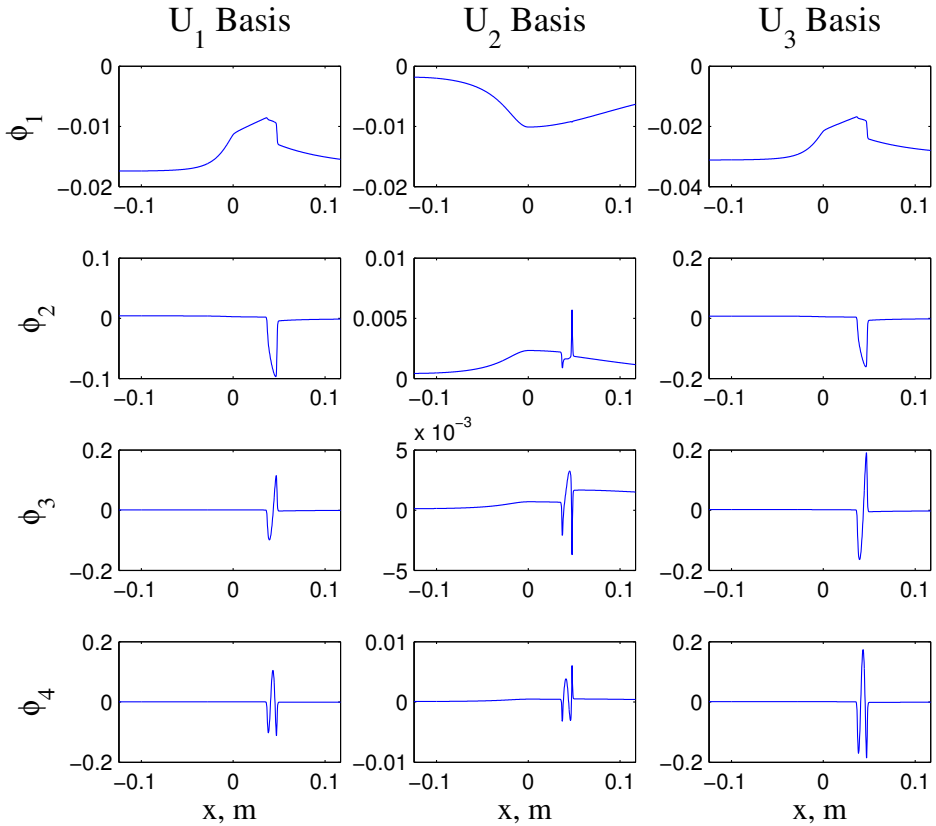

(b)

Figure 10: (a) Singular values of snapshot matrix for the case of a ramp decrease in $p_{e} / p_{t, i}$ from 0.87 to 0.85 over 0.01 seconds. (b) First four basis vectors $\left(\phi_{1}, \phi_{2}, \phi_{3}, \phi_{4}\right)$ as a function of $x$ for each of the three conservation variables $\left(U_{1}, U_{2}, U_{3}\right)$ in the case of a ramp decrease in $p_{e} / p_{t, i}$ from 0.87 to 0.85 .

In the reduced-order model, Eq. (5) is solved for the POD coeffiecients $a_{j}, j=1, \ldots, M_{L}$ versus time using 40 basis vectors (significantly more compared to the fully-expanded nozzle example, where three basis 
vectors was sufficient). The first four POD coefficients versus time are shown in Fig. 11 . Figure 12 shows the corresponding solution in time reconstructed from the POD coefficients in Fig. 11 and the basis vectors in Fig. 10b using Eq. (3). By keeping only the first 40 modes, the CFL number can be increased from 2.5 to 8 in this case resulting in a speed-up factor of approximatly 2.4 .
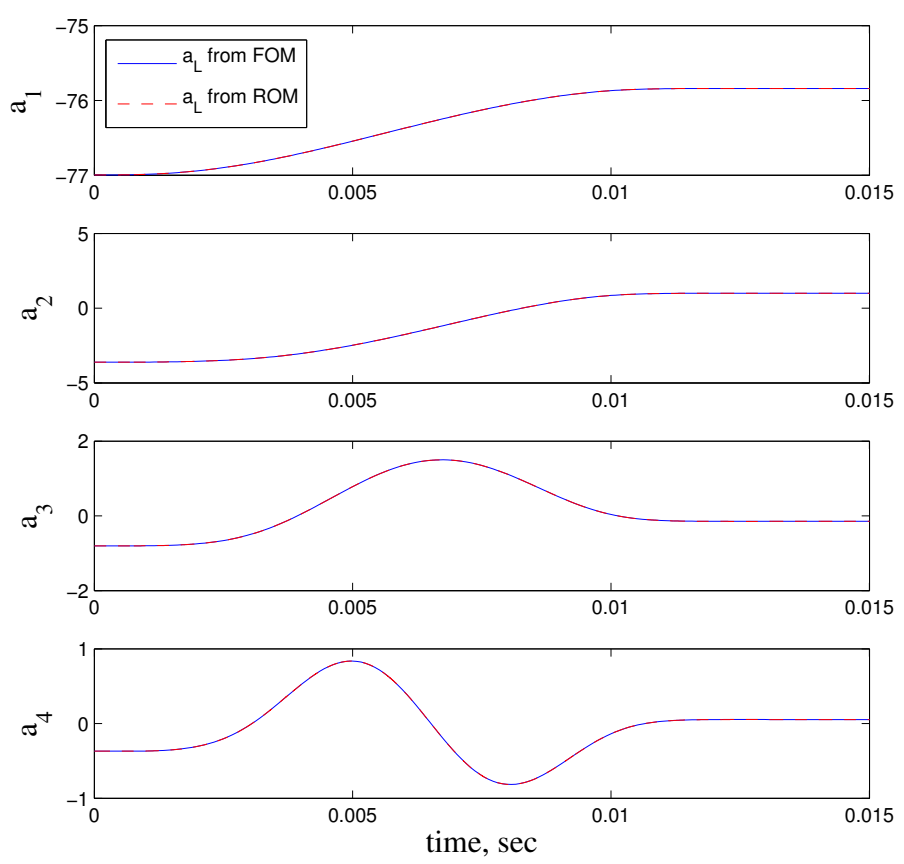

Figure 11: First four POD coefficients $\left(a_{1,2,3,4}\right)$ versus time from the reduced-order model (using 40 basis vectors and increasing CFL to 8) along with the corresponding POD coefficients required to reproduce the full-order solution for comparison. 

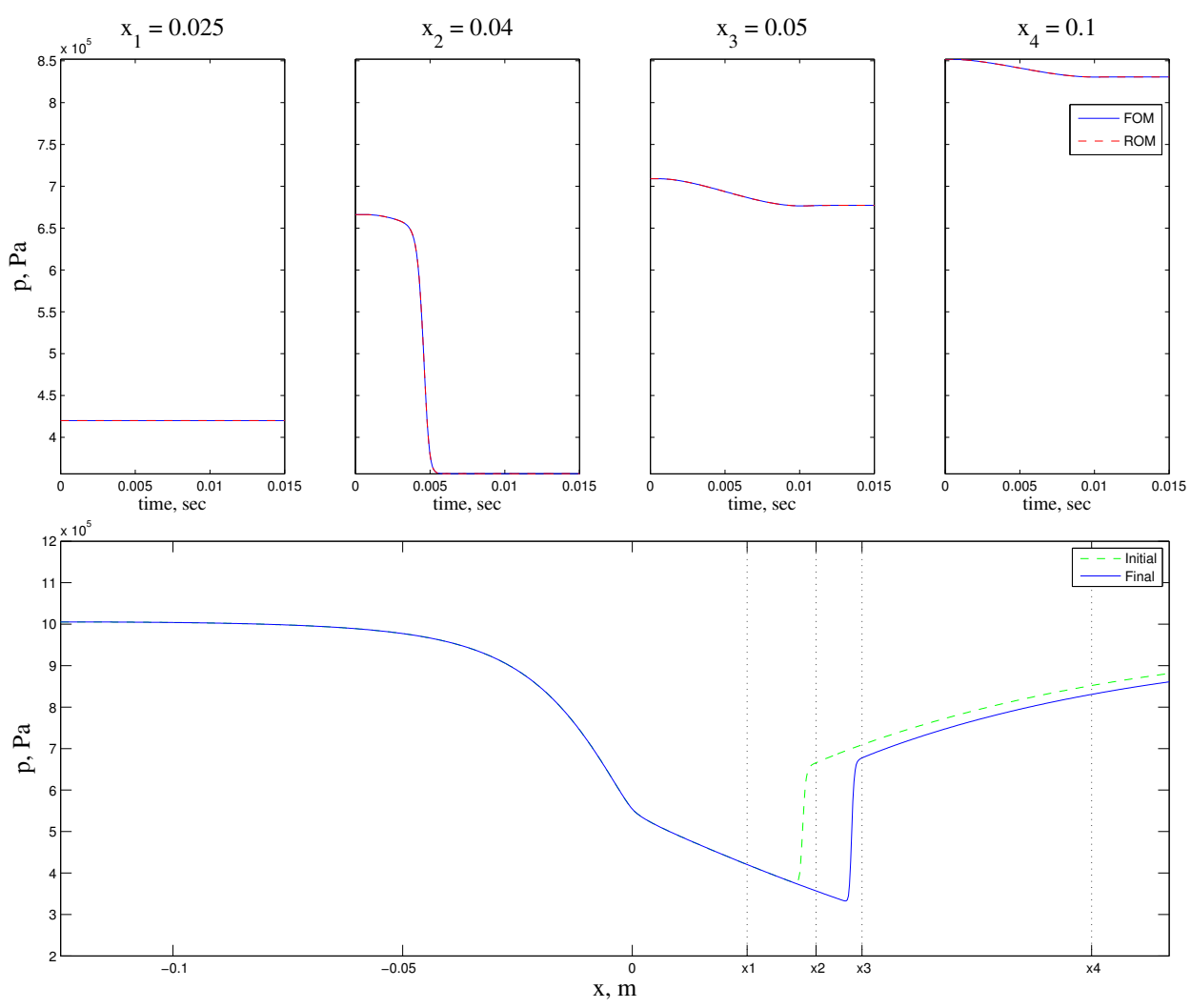

Figure 12: Pressure (reconstructed from the reduced-order model POD coefficients) versus time at four locations in the nozzle for $M_{L}=40$. Also displayed for comparison is the pressure from the full-order solution.

To further increase the speed-up factor, nonlinear acceleration utilizing DEIM and L2-Norm Minimization is again applied. The residuals from the unsteady, full-order model are compiled into a snapshot matrix and a singular value decomposition is performed on the matrix. For this example, demonstrating nonlinear acceleration, 27 basis vectors are used. Using the first 27 modes from the residual space, the DEIM algorithm selects the 27 sampling locations seen in Fig. 13. Note that the sampling point locations are clustered around the shock region. To implement the DEIM technique, Eqs. (7) and (8) are used to reconstruct the full residual from the residuals calculated at the sampling locations, the results of which are shown in Fig. 13a at four different times for the energy-equation residual. Using DEIM acceleration, the speed-up factor compared to the full-order model is approximately 4.3. The methods in Section III for estimating the residual using L2-Norm Minimization (Eqs. (9) and (11)) are also applied to this case and the energy-equation residual at the same four times as the DEIM case is shown in Fig. 13b for comparison. 

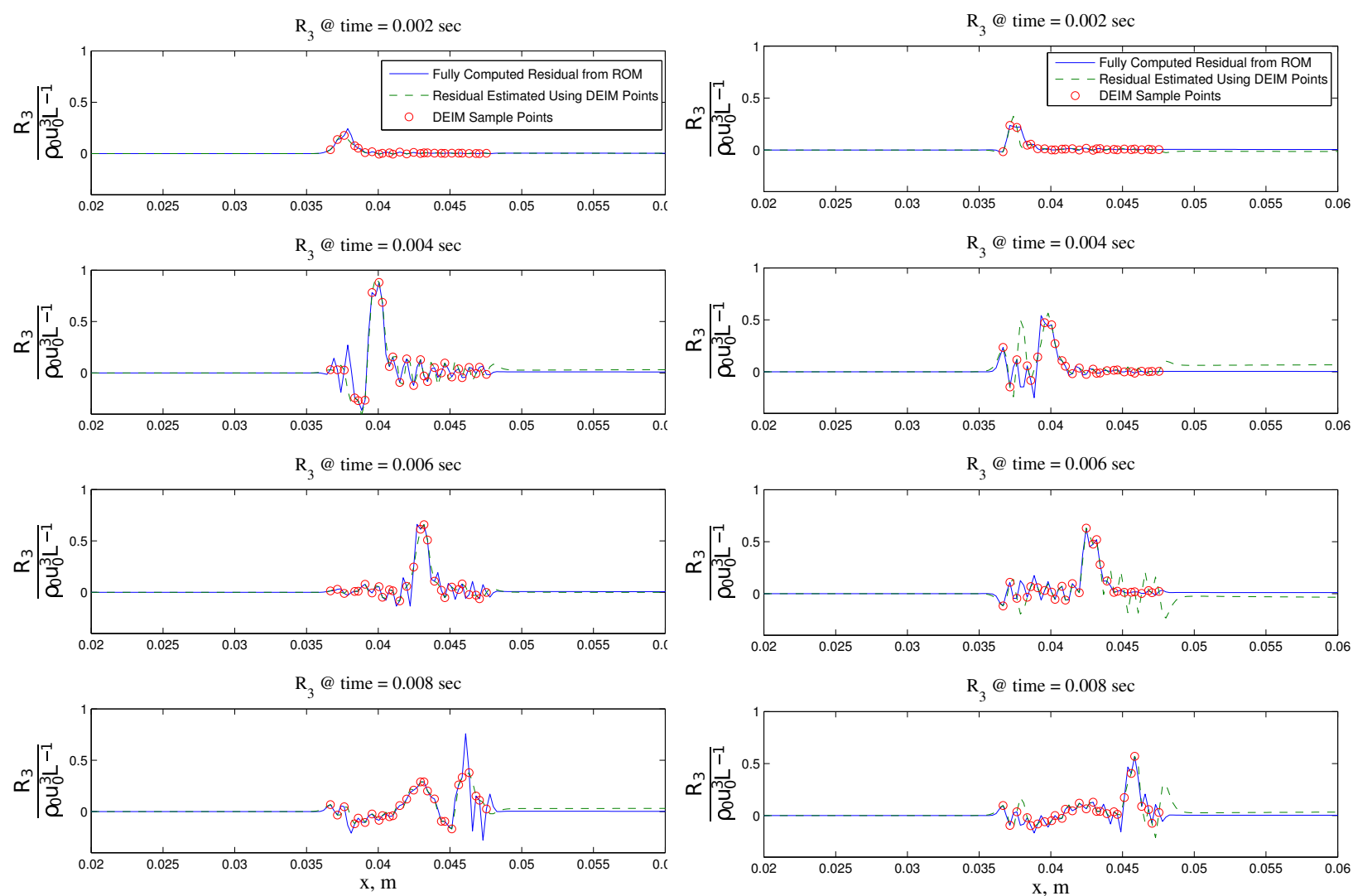

(a) Residual reconstruction using DEIM.

(b) Residual reconstruction using L2-Norm.

Figure 13: Comparison of the fully computed energy-equation residual $R_{3}$ to the residual calculated from (a) DEIM and (b) L2-Norm Minimization. The 27 sample point locations are calculated using the DEIM algorithm and are the same for both cases.

While the conventional nonlinear acceleration technique using DEIM shows promise for further decreasing the computational time of ROMs, stability remains an issue. To investigate the stability of the ROM using acceleration, the eigenvalues of the Jacobian matrix $d R(U) / d U$ are plotted for different reducedorder modeling techniques, each of which contain the same number of basis vectors. Figure 14a shows the eigenvalues for the reduced-order model without acceleration and using 28 basis vectors. Note that all the eigenvalues have negative real parts. The eigenvalues of the full-order model are nearly identical to those of the reduced-order model in Fig. 14a; both the full-order model and the reduced-order model without acceleration are stable. When DEIM acceleration is applied however, most of the eigenvalues collapse to zero and the real part of some eigenvalues become positive as seen in Fig. 14b. The model is unstable using DEIM acceleration with 28 basis vectors. However, as was the case in the previous example of a fully-expanded nozzle, using L2-Norm Minimization instead of DEIM stabilizes the reduced-order model. The eigenvalues of the Jacobian matrix for the ROM using L2-Norm Minimization are shown in Fig. 15 and all the eigenvalues have real parts less than or equal to zero. 


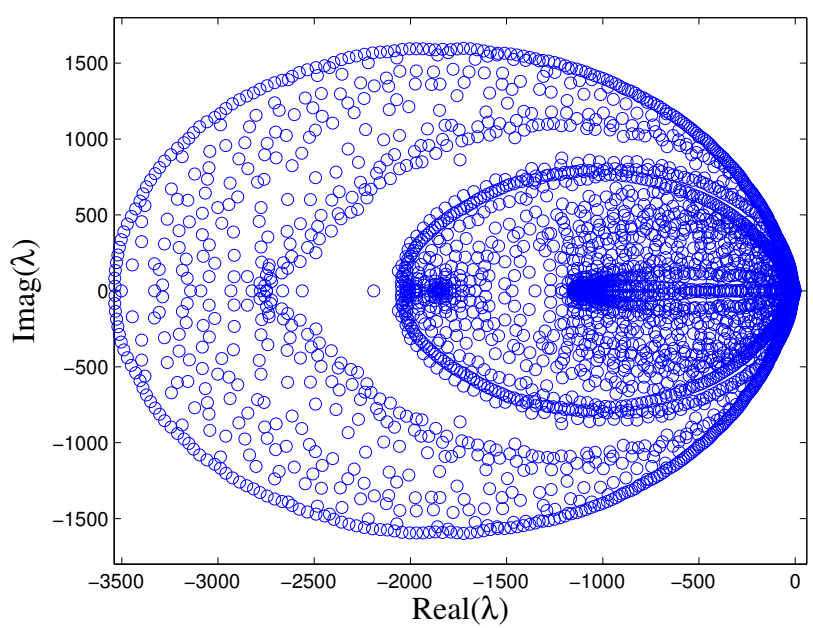

(a) Without acceleration.

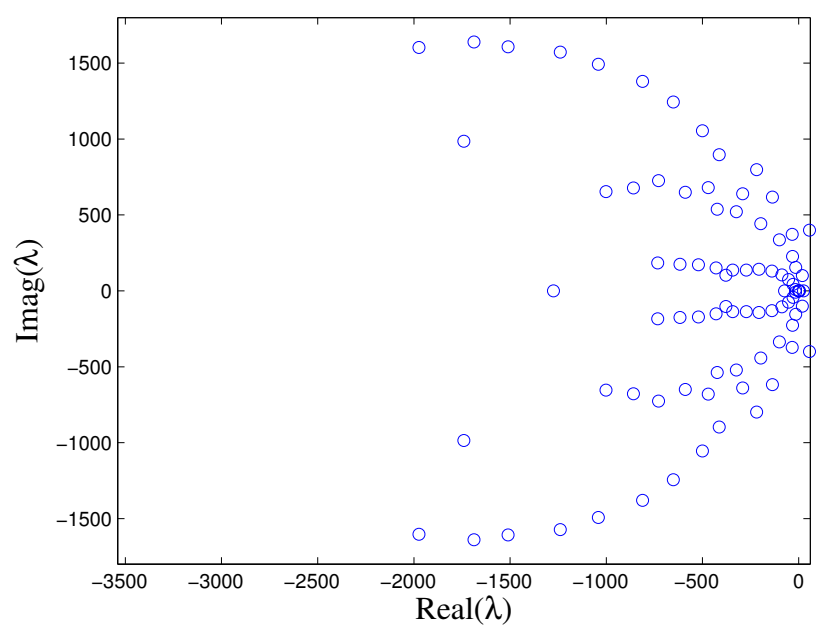

(b) Acceleration using DEIM.

Figure 14: Eigenvalues of the Jacobian matrix $d R(U) / d U$ from the reduced-order model (a) without acceleration and (b) employing DEIM for acceleration. ( $M_{L}=\mathbf{2 8}$ for both cases.)

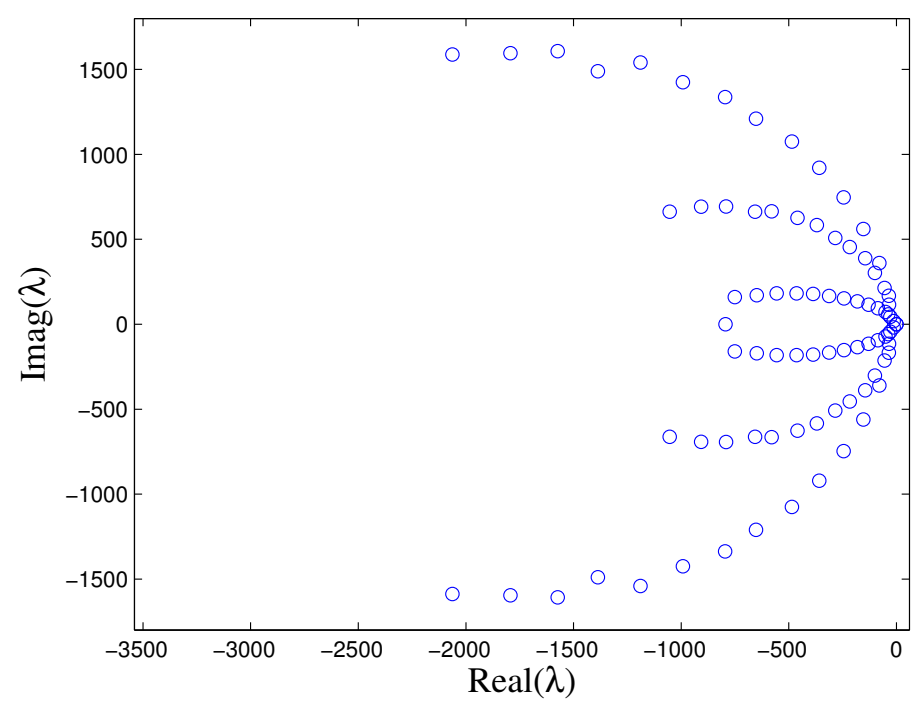

Figure 15: Eigenvalues of the Jacobian matrix $d R(U) / d U$ from the reduced-order model employing L2-Norm Minimization for acceleration and using 28 basis vectors.

Again, to further assess the stability of the reduced-order model, the global eigenvectors are compared for the different models. The global eigenvectors for the full-order model are identical to the global eigenvectors for the reduced-order model without acceleration. Figure 16 compares the global eigenvectors of the ROM employing DEIM and L2-Norm Minimization to the global eigenvectors of the ROM without acceleration. Note that there is significant improvement for eigenvectors corresponding to all three conservation variables using L2-Norm Minimization over the conventional DEIM approach. 

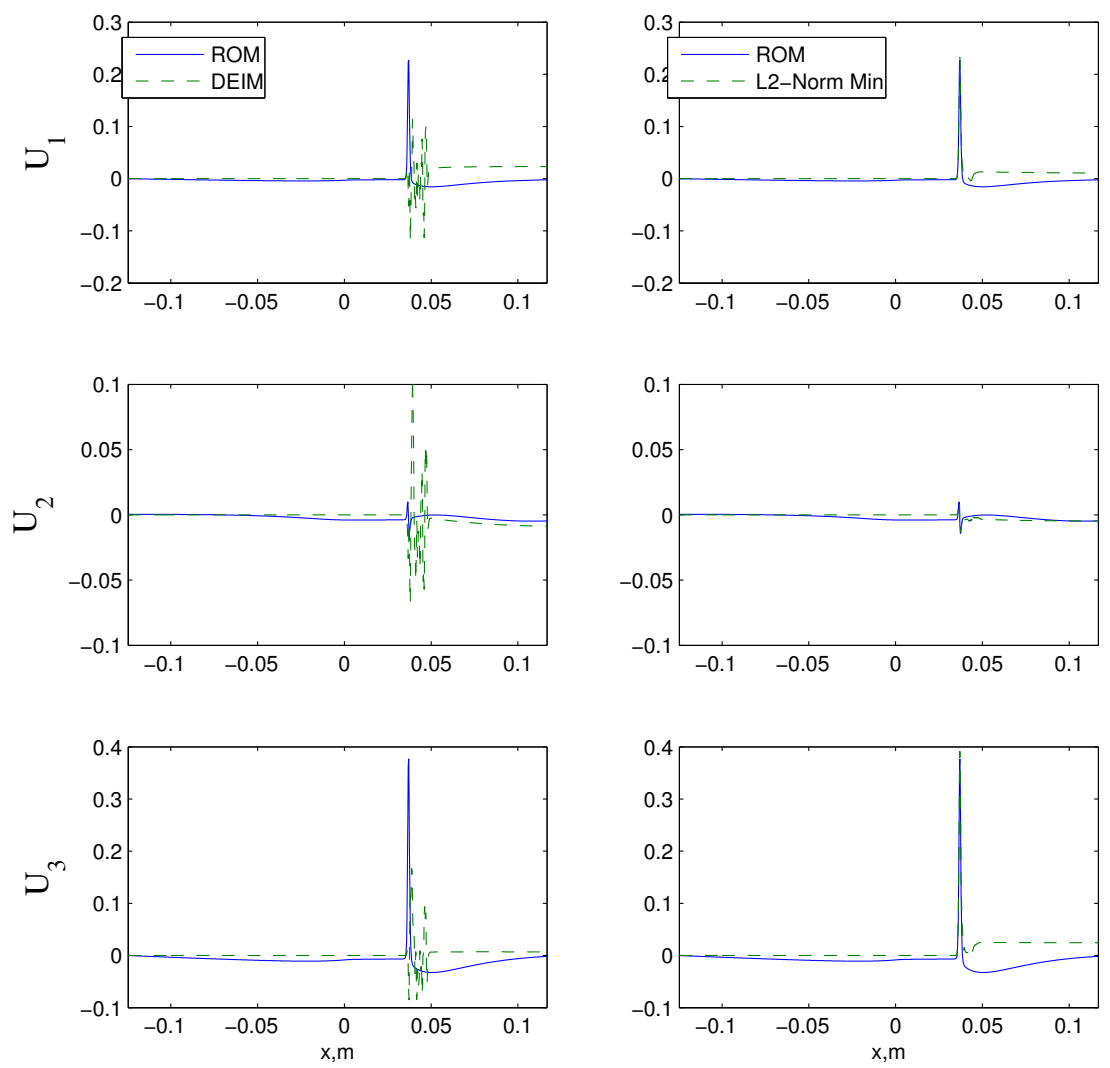

Figure 16: Global eigenvectors of the reduced-order model without acceleration compared to the reduced-order model employing DEIM acceleration in the left column and L2-Norm Minimization acceleration in the right column.

\section{Conclusion}

Preventing, or at least managing, engine unstart in high-speed, air-breathing propulsion systems remains an immense challenge. As engineers seek to model more complex phenomena, such as engine unstart, in earlier design stages, improved modeling techniques are required. POD/Galerkin projection-based reduced-order models have been successfully applied to linearized flow problems. Engine unstart, however, is characterized by large shock motion and is highly nonlinear. To assess the performance and stability of POD/Galerkin projection-based reduced-order models when applied to nonlinear flows, two nonlinear flow problems were studied: (1) a fully-expanded nozzle flow where the inlet stagnation pressure is increase from 5 to 10 atm over 0.01 seconds and (2) a nozzle flow with a shock in diverging section where the nozzle pressure ratio is decreased from 0.85 to 0.87 over 0.01 seconds. In both examples, the POD/Galerkin projection-based reduced order model produced stable results with some degree of computational speed-up. However, the degree of speed up was significantly smaller in the case with a shock wave. The discontinuity requires a large number of basis vectors which limits the allowed time step increase, reducing the computational speed-up.

The nonlinear governing equations usually require a full function evaluation of the residual terms at each time step. To circumvent this constraint, the Discrete Empirical Interpolation Method (DEIM), a nonlinear acceleration technique, was applied in both cases. The DEIM was found to have poor stability characteristics in a number of test runs and thus an alternate acceleration technique based on L2-Norm Minimization was proposed. The L2-Norm minimization is similar - in principle - to the Gappy POD technique.

For both problems, instances were found where the reduced-order model employing the conventional DEIM technique was unstable but became stable, with minimal trade-off in accuracy, when L2-Norm Mini- 
mization was instead applied. The eigenvalues of the Jacobian matrix for the reduced-order model employing DEIM and L2-Norm Minimization were plotted and showed further evidence for the latter's improved stability over DEIM. The improvement in stability can be attributed to the smoother residual field that is not fit to every sample point. With the reduced order-model employing L2-Norm Minimization, up to an order of magnitude cost reduction over the full-order model was achievable even for problems with strong moving shocks. Continuing research is being directed towards optimal selection of sample points for acceleration and in exploring the utility of compressed sensing approaches.

\section{Acknowledgments}

The authors thank Prof. James Driscoll of the University of Michigan for his technical advise. The authors would like to acknowledge the Control Science Center of Excellence at the Air Force Research Laboratory in Dayton, OH for funding this research.

\section{References} 2005.

${ }^{1}$ Conway, E., High-Speed Dreams: NASA and the Technopolitics of Supersonic Transportation, 1945-1999, JHU Press,

${ }^{2}$ Segal, C., The scramjet engine: processes and characteristics, Vol. 25, Cambridge University Press, 2009.

${ }^{3}$ Bolender, M. A. and Doman, D. B., "Nonlinear longitudinal dynamical model of an air-breathing hypersonic vehicle," Journal of Spacecraft and Rockets, Vol. 44, No. 2, 2007, pp. 374-387.

${ }^{4}$ Dalle, D. J., Fotia, M. L., and Driscoll, J. F., "Reduced-Order Modeling of Two-Dimensional Supersonic Flows with Applications to Scramjet Inlets," Journal of Propulsion and Power, Vol. 26, No. 3, 2010, pp. 545-555.

${ }^{5}$ Torrez, S. M., Driscoll, J. F., Ihme, M., and Fotia, M. L., "Reduced-order modeling of turbulent reacting flows with application to ramjets and scramjets," Journal of propulsion and power, Vol. 27, No. 2, 2011, pp. 371-382.

${ }^{6}$ Torrez, S. M., Dalle, D. J., and Driscoll, J. F., "New Method for Computing Performance of Choked Reacting Flows and Ram-to-Scram Transition," Journal of Propulsion and Power, Vol. 29, No. 2, 2013, pp. 433-445.

${ }^{7}$ Dalle, D., Torrez, S. M., and Driscoll, J. F., "Rapid Analysis of Scramjet and Linear Plug Nozzles," Journal of Propulsion and Power, Vol. 28, No. 3, 2012, pp. 545-555.

${ }^{8}$ Dalle, D. D. and Driscoll, J. F., "Ascent Trajectories of Hypersonic Aircraft: Operability Limits Due to Engine Unstart," Journal of Aircrafs, Under Review.

${ }^{9}$ Lucia, D. J., King, P. I., and Beran, P. S., "Reduced order modeling of a two-dimensional flow with moving shocks," Computers 8 fluids, Vol. 32, No. 7, 2003, pp. 917-938.

${ }^{10}$ Yamaleev, N. K. and Pathak, K. A., "Nonlinear model reduction for unsteady discontinuous flows," Journal of Computational Physics, Vol. 245, 2013, pp. 1-13.

${ }^{11}$ Chaturantabut, S. and Sorensen, D. C., "Discrete empirical interpolation for nonlinear model reduction," Decision and Control, 2009 held jointly with the 2009 28th Chinese Control Conference. CDC/CCC 2009. Proceedings of the 48th IEEE Conference on, IEEE, 2009, pp. 4316-4321.

${ }^{12}$ Hutzel, J. R., Decker, D. D., and Donbar, J. M., "Scramjet isolator shock-train leading-edge location modeling," AIAA Paper, Vol. 2223, 2011.

${ }^{13}$ MacMartin, D. G., "Dynamics and control of shock motion in a near-isentropic inlet," Journal of Aircraft, Vol. 41, No. 4, 2004, pp. 846-853.

${ }^{14}$ Chicatelli, A. and Hartley, T. T., A Method for Generating Reduced-Order Linear Models of Multidimensional Supersonic Inlets, National Aeronautics and Space Administration, Lewis Research Center, 1998.

${ }^{15}$ Mayer, D. W. and Paynter, G. C., "Prediction of supersonic inlet unstart caused by freestream disturbances," AIAA journal, Vol. 33, No. 2, 1995, pp. 266-275.

${ }^{16}$ Holmes, P., Lumley, J. L., and Berkooz, G., Turbulence, coherent structures, dynamical systems and symmetry, Cambridge university press, 1998.

${ }^{17}$ Rowley, C. W., Colonius, T., and Murray, R. M., "Model reduction for compressible flows using POD and Galerkin projection," Physica D: Nonlinear Phenomena, Vol. 189, No. 1, 2004, pp. 115-129.

${ }^{18}$ Bui-Thanh, T., Damodaran, M., and Willcox, K. E., "Aerodynamic data reconstruction and inverse design using proper orthogonal decomposition," AIAA journal, Vol. 42, No. 8, 2004, pp. 1505-1516.

${ }^{19}$ Johnson, A. D. and Papamoschou, D., "Instability of shock-induced nozzle flow separation," Physics of Fluids (1994present), Vol. 22, No. 1, 2010, pp. 016102.

${ }^{20}$ Mandal, J. and Deshpande, S., "Kinetic flux vector splitting for Euler equations," Computers 83 fluids, Vol. 23, No. 2, 1994, pp. $447-478$. 